ISSN 0103-9954

\title{
INFLUÊNCIA DE VARIÁVEIS AMBIENTAIS SOBRE O PADRÃO ESTRUTURAL E FLORÍSTICO DO COMPONENTE ARBÓREO, EM UM FRAGMENTO DE FLORESTA OMBRÓFILA MISTA MONTANA EM LAGES, SC
}

\section{INFLUENCE OF ENVIRONMENTAL VARIABLES ON THE TREE COMMUNITY STRUCTURE AND FLORISTIC PATTERNS IN A MONTANE ARAUCARIA FOREST FRAGMENT IN LAGES, SANTA CATARINA STATE}

Pedro Higuchi ${ }^{1}$ Ana Carolina da Silva ${ }^{2}$ Tiago de Souza Ferreira ${ }^{3}$ Sheila Trierveiler de Souza ${ }^{3}$ Juliano Pereira Gomes ${ }^{4}$ Karina Montibeller da Silva $^{5}$ Kristiana Fiorentin dos Santos ${ }^{3}$ Caroline Linke ${ }^{3}$ Patrícia da Silva Paulino ${ }^{3}$

\section{RESUMO}

O objetivo deste trabalho foi caracterizar a composição florística e a estrutura do componente arbóreo de um fragmento de Floresta Ombrófila Mista Montana e avaliar a influência de variáveis ambientais sobre os padrões encontrados. A área de estudo situa-se no município de Lages, SC, às margens do Rio Caveiras $\left(27^{\circ} 51^{\prime} 19.20^{\prime \prime} \mathrm{S}\right.$ e $\left.50^{\circ} 10^{\prime} 33,39^{\prime \prime} \mathrm{W}\right)$. O levantamento da vegetação e das variáveis ambientais (características químicas e físicas dos solos, relevo e cobertura do dossel) foi realizado em 50 parcelas permanentes de 20x10 $\mathrm{m}$, alocadas de forma sistemática estratificada no fragmento. Todas as árvores vivas com circunferência $\geq$ $15,7 \mathrm{~cm}$ (medidas a 1,30 m do solo - CAP) foram mensuradas (CAP e altura) e identificadas. Foi calculado o valor de importância (VI) das espécies encontradas, realizada a ordenação das parcelas em função da abundância das espécies, por meio da analise de NMDS (Nonmetric multidimensional scalling) e plotadas a posteriori as variáveis ambientais significativas no diagrama de ordenação. Foram amostrados 1.843 indivíduos, que totalizaram uma área basal de $36,45 \mathrm{~m}^{2} /$ ha, distribuídos em 37 famílias botânicas, 63 gêneros e 92 espécies. As três espécies com os maiores valores de VI foram Araucaria angustifolia (Bertol.) Kuntze, Lithraea brasiliensis Marchand e Jacaranda puberula Cham. As variáveis que apresentaram maior correlação com a estrutura e a composição florística do componente arbóreo foram o pH, o desnível máximo da parcela e o teor de $\mathrm{Mg}$.

Palavras-chave: heterogeneidade ambiental; floresta de araucária; análise multivariada.

\section{ABSTRACT}

This study aimed to characterize the floristic composition and structure of the tree component of a montane Araucaria Forest fragment and to assess the influence of environmental variables on the patterns observed. The study area was located in the municipality of Lages, Santa Catarina state, close to the margin of the Caveiras River. The vegetation and the environmental variables (physical and chemical soils characteristics, relief and canopy cover) were surveyed within $50,20 \times 10 \mathrm{~m}$, permanent plots allocated systematically stratified

1. Engenheiro Florestal, Dr., Professor Adjunto do Departamento de Engenharia Florestal, Centro de Ciências Agroveterinárias, Universidade do Estado de Santa Catarina, Av Luiz de Camões, 2090, Bairro Conta Dinheiro, CEP 88520-000, Lages (SC). higuchip@gmail.com

2. Engenheira Florestal, Dr ${ }^{\mathrm{a}}$., Professora Adjunto do Departamento de Engenharia Florestal, Centro de Ciências Agroveterinárias, Universidade do Estado de Santa Catarina, Av Luiz de Camões, 2090, Bairro Conta Dinheiro, CEP 88520-000, Lages (SC). carol_sil4@yahoo.com.br

3. Estudante, Curso de Engenharia Florestal, Centro de Ciências Agroveterinárias, Universidade do Estado de Santa Catarina, Av Luiz de Camões, 2090, Bairro Conta Dinheiro, CEP 88520-000, Lages (SC). Bolsista PROBIC

4. Engenheiro Florestal, Mestrando do Programa de Pós-graduação em Produção Vegetal, Centro de Ciências Agroveterinárias, Universidade do Estado de Santa Catarina, Av Luiz de Camões, 2090, Bairro Conta Dinheiro, CEP 88520-000, Lages (SC).julianopgomes@yahoo.com.br

5. Engenheira Florestal, Mestrando do Programa de Pós-graduação em Produção Vegetal, Centro de Ciências Agroveterinárias, Universidade do Estado de Santa Catarina, Av Luiz de Camões, 2090, Bairro Conta Dinheiro, CEP 88520-000, Lages (SC). karynaflorestal@yahoo.com.br

Recebido para publicação em 23/04/2010 e aceito em 18/03/2010 
Higuchi, P. et al.

in the forest fragment. All trees with circumference at breast height $(\mathrm{CBH}) \geq 15,7 \mathrm{~cm}$ were measured $(\mathrm{CBH}$ and height) and identified. The importance values (IV) of the found species were calculated, the plots and species were ordinated by an analysis of NMDS (Nonmetric Multidimensional Scaling), according to the abundance of species, and the significant environmental variables plotted "a posteriori" in the ordination diagram. One thousand, eight hundred and forty-three $(1,843)$ individuals were sampled, which added up to a total basal area of $36.45 \mathrm{~m}^{2}$, distributed in 37 botanical families, 63 genera and 92 species. The three species with the highest values of VI were Araucaria angustifolia (Bertol.) Kuntze, Lithraea brasiliensis Marchand and Jacaranda puberula Cham. The variables that showed the higher correlation with the tree component structure and floristic composition were the $\mathrm{pH}$, the plot highest declivity and the $\mathrm{Mg}$ content.

Keywords: environmental heterogeneity; araucaria forest; multivariate analysis.

\section{INTRODUÇÃO}

A Floresta de Araucária, classificada como Floresta Ombrófila Mista (FOM), de acordo com o IBGE (1992), é uma das principais formações vegetacionais da região sul do Brasil. Sua maior área de distribuição geográfica está associada aos locais de elevada altitude e com baixas temperaturas médias anuais, ocorrendo, de forma associada aos campos naturais, predominantemente na região do planalto meridional.

Como a maior parte das florestas do domínio atlântico, essas foram, e são, intensamente perturbadas e fragmentadas. Durante o ciclo da madeira, nas décadas de 50 e 60, ocorreu intensa exploração de espécies de interesse madeireiro, como da Araucária (Araucaria angustifolia (Bertol.) Kuntze) e da Imbuia (Ocotea porosa (Mez) L.Barroso), e não madeireiro, como do Xaxim (Dicksonia sellowiana Hook.). Outra perturbação recorrente é a utilização de remanescentes de FOM para a criação de gado, pois o interior dos fragmentos florestais serve como abrigo e fonte de alimentos para os animais durante os períodos desfavoráveis, como no inverno. Além disso, a necessidade de áreas para o desenvolvimento de atividades agrícolas e pecuárias aumentou a pressão sobre a FOM, reduzindo a área original a fragmentos florestais de diferentes formas, tamanhos e níveis de perturbação. Apesar de não existirem trabalhos que demonstrem estes efeitos, provavelmente a composição florística e a estrutura da FOM foram alteradas, em função desse histórico de perturbação.

No entanto, a conservação e a restauração dos remanescentes de FOMsãojustificáveis e necessárias, pois estes ainda são de grande importância ambiental e social. Essas áreas desempenham funções ambientais como, por exemplo, a proteção do solo e a manutenção dos cursos de água; podem sequestrar o carbono atmosférico, mitigando os efeitos negativos das mudanças climáticas; e, no contexto de ecologia da paisagem, podem permitir uma maior conexão entre fragmentos e reservas florestais, funcionando como stepping stones e corredores ecológicos para espécies animais e vegetais. Do ponto de vista social, muitas comunidades rurais têm a FOM como uma importante fonte de renda, por meio da extração da semente da araucária, conhecida popularmente como pinhão, e de outros produtos madeireiros e não madereiros. Para a conservação desses remanescentes é necessária uma correta orientação técnica a essas comunidades, objetivando evitar a exploração não sustentável dos recursos florestais, o que garantiria a manutenção do ecossistema.

Com o propósito de subsidiar estratégias de conservação e restauração nas áreas de FOM, é fundamental um maior conhecimento sobre os padrões florísticos e estruturais das florestas, além do entendimento da influência de variáveis ambientais sobre esses padrões. Tais estudos são importantes, pois fornecem informações a respeito dos microssítios de ocorrência das espécies arbóreas, o que pode facilitar a indicação destas para o plantio em condições ambientais específicas.

Vários estudos no Brasil têm demonstrado que comunidades arbóreas, em ecossistemas florestais fragmentados, apresentam forte variação espacial em função da heterogeneidade ambiental, provocada por diferentes condições edáficas (RODRIGUES et al., 2007; HIGUCHI et al., 2008a; SILVA et al., 2009; SILVA et al., 2010), de relevo (ESPÍRITO-SANTO et al., 2002), de luminosidade associadas a bordas e clareiras (CARDOSO e SCHIAVINI, 2002; OLIVEIRA FILHO et al., 2007) e do histórico de perturbação (OLIVEIRA FILHO et al., 1997; HIGUCHI et al., 2008b). No estado de Santa Catarina, são raros os trabalhos qualiquantitativos publicados que investigam a relação existente entre o componente arbóreo da FOM e 
as variáveis ambientais (e.g. PUCHALSKI, 2004). Desta forma, o presente estudo teve como propostas: I) caracterizar a composição florística e a estrutura do componente arbóreo de um fragmento de FOM na região de Lages, Santa Catarina e, II) avaliar a influência de variáveis edáficas, topográficas e de cobertura do dossel sobre a florística e a estrutura da vegetação arbórea. A hipótese a ser testada é a de que o componente arbóreo apresenta variações florísticas e estruturais associadas à heterogeneidade ambiental existente.

\section{MATERIAL E MÉTODO}

\section{Caracterização da área de estudo}

$\mathrm{O}$ presente estudo foi realizado em um fragmento de Floresta Ombrófila Mista Montana, com área aproximada de 103,06 ha e altitude média de $990 \mathrm{~m}$ (altitude máxima $=1.036 \mathrm{~m}$ e altitude mínima $=901 \mathrm{~m})$, localizado no município de Lages, SC, nas margens do Rio Caveiras (2751'19.20'S e $50^{\circ} 10^{\prime} 33,39^{\prime \prime} \mathrm{W}$ ) (Figura 1). De acordo com a estação meteorológica de Lages, administrada pela EPAGRI/CIRAM/INMET, a precipitação e a temperatura média anual (1970-2010) na região do fragmento é de, respectivamente, $1.682,80 \mathrm{~mm}$ e $15,9^{\circ} \mathrm{C}$.

\section{Coleta de dados}

Para o levantamento das variáveis ambientais, da composição e da estrutura do componente arbóreo, foram alocadas 50 parcelas permanentes de 10x20 $\mathrm{m}\left(20 \mathrm{~m}^{2}\right)$, totalizando 1 ha de área amostrada. As parcelas foram distribuídas ao longo de transectos, distanciadas $30 \mathrm{~m}$ entre si dentro de cada transecto (Figura 2). Esta distribuição

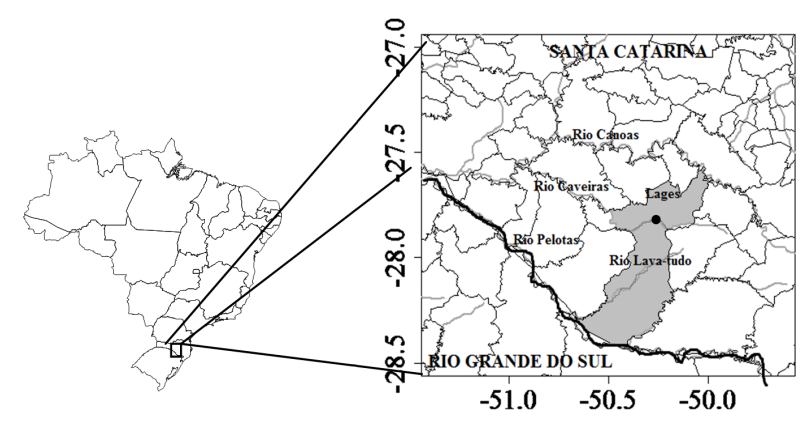

FIGURA 1: Localização geográfica do fragmento de Floresta Ombrófila Mista Montana estudado em Lages, Santa Catarina.

FIGURE 1: Geographical location of the study montane Araucaria forest fragment in Lages, Santa Catarina state. espacial foi definida com a finalidade de amostrar adequadamente as variações ambientais, tais como borda $\times$ interior e gradientes de topografia.

Em cada parcela permanente, todos os indivíduos arbóreos que apresentaram CAP (circunferência à altura do peito, medida a $1,30 \mathrm{~m}$ do solo) igual ou superior a $15,7 \mathrm{~cm}$ foram identificados e marcados com plaquetas de alumínio. Em cada um destes indivíduos foi medido o CAP, com fita métrica, e estimada a altura. Indivíduos com troncos múltiplos foram medidos quando a raiz da soma dos quadrados dos CAPs foi maior do que $15,7 \mathrm{~cm}$. Não foram incluídos indivíduos mortos e lianas. Para complementar a lista florística, foram realizados caminhamentos e feitas coletas de indivíduos arbóreos fora das parcelas. As identificações foram realizadas por meio de pareceres de especialistas e literatura especializada. As espécies foram classificadas nas famílias de acordo com o sistema APG III (APG III, 2009).

Foi realizado um levantamento topográfico em cada parcela, com auxílio de trena, bússola, clinômetro e GPS, a partir do qual foram definidas três variáveis referentes ao relevo (OLIVEIRA FILHO et al., 1994): cota média (m), desnível máximo (m) e declividade média (m). A cota média foi considerada como a média das cotas dos quatro vértices de cada parcela. O desnível máximo correspondeu à maior distância vertical entre os vértices das parcelas. A declividade média foi calculada por meio da média simples das declividades dos quatro lados das parcelas. As propriedades químicas e físicas dos solos, de cada parcela, foram obtidas por meio da análise de amostras compostas superficiais, provenientes de quatro coletas distribuídas em cada parcela, a uma profundidade 0 a $20 \mathrm{~cm}$. As análises foram realizadas no Laboratório de Solos do Centro de Ciências Agroveterinárias da Universidade do Estado de Santa Catarina (CAV/UDESC), de acordo com os procedimentos recomendados pela EMBRAPA (1997). Foram quantificados o $\mathrm{pH}$, fósforo, potássio, cálcio, magnésio, sódio, alumínio e porcentagem de argila, e calculados os índices: $\mathrm{H}+\mathrm{Al}, \mathrm{t}$ (CTC efetiva), $\mathrm{T}(\mathrm{CTC}$ a $\mathrm{pH}=$ 7,0), SB (soma de bases trocáveis) e V (saturação de bases da CTC a pH =7,0). A avaliação da cobertura do dossel foi realizada por meio da média de quatro leituras, em direção ao norte, sul, leste e oeste, no centro de cada parcela, utilizando um densiômetro esférico (modelo A) côncavo (LEMMON, 1956), no período de novembro a dezembro de 2009. 


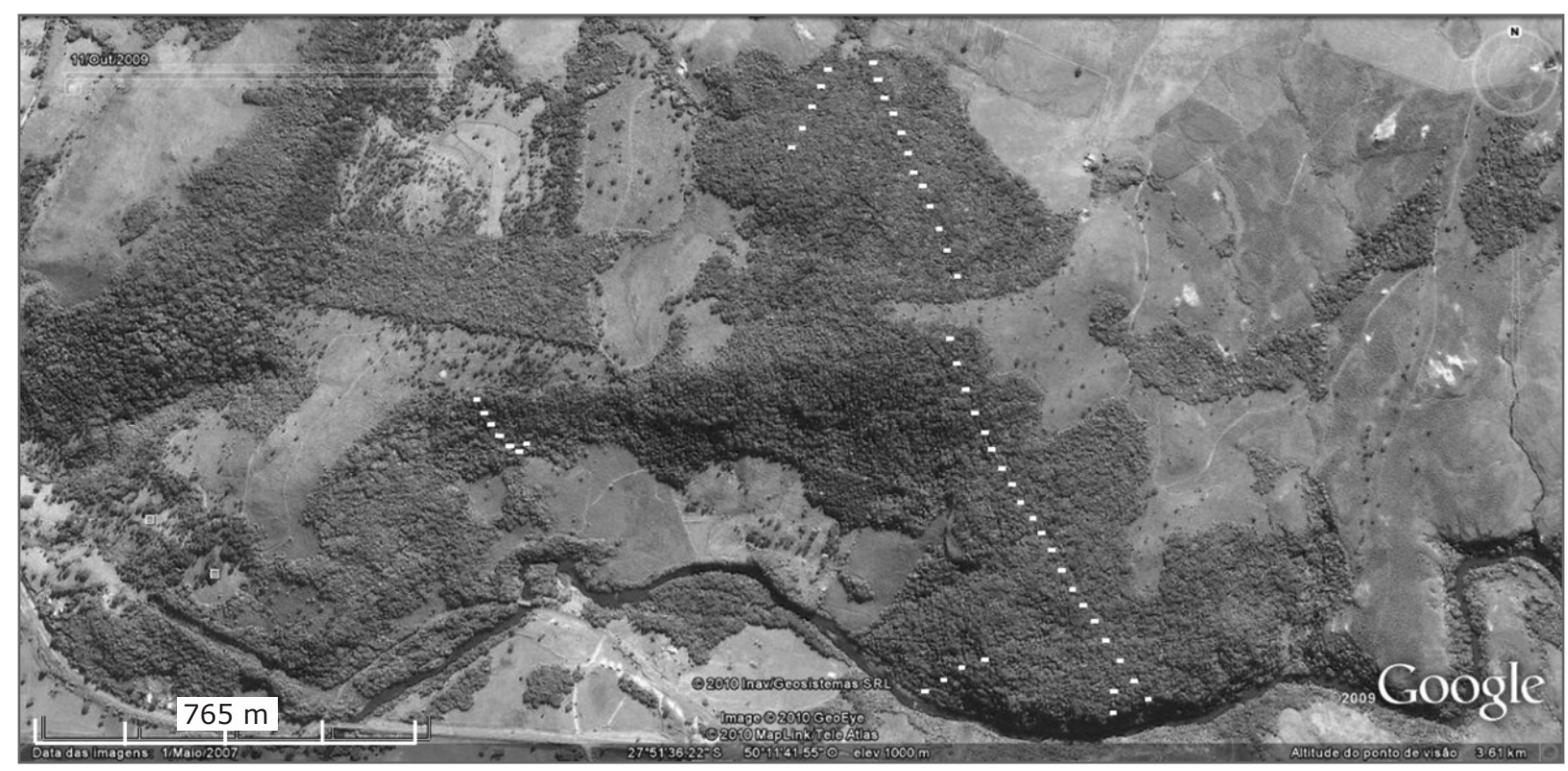

FIGURA 2: Localização geográfica das parcelas amostradas (retângulos em branco) no fragmento de Floresta Ombrófila Mista Montana estudado em Lages, Santa Catarina. Fonte: Google Earth

FIGURE 2: Sample plots geographical location (blank rectangles) in the study of a montane Araucaria forest fragment in Lages, Santa Catarina. Source: Google Earth.

\section{Análise dos dados}

Para a quantificação da diversidade de espécies arbóreas e dominância ecológica, foram calculados, respectivamente, o índice de Shannon $\left(\mathrm{H}^{\prime}\right)$ e a equabilidade de Pielou (J') (BROWER et al., 1990). Foram extraídos, para as amostras, os estimadores de Jackknife de primeira e segunda ordem (HELTSHE e FORRESTER, 1983).

A estrutura horizontal da comunidade arbórea foi descrita a partir do cálculo, para cada espécie, dos parâmetros quantitativos clássicos propostos por Mueller-Dombois e Ellemberg (1974): densidade absoluta, frequência absoluta, dominância absoluta expressa pela área basal, densidade relativa, frequência relativa, dominância relativa e valor de importância (VI).

Para verificar a variação ambiental, entre as parcelas estudadas, foi produzida uma ordenação ambiental das parcelas, a partir das variáveis estudadas, por meio da análise dos componentes principais (PCA). Também foi feita a ordenação das parcelas em função das características estruturais e florísticas, realizada por meio da técnica de análise multivariada NMDS (Nonmetric multidimensional scalling), empregando quatro dimensões $(\mathrm{k}=4)$ (MINCHIN, 1987). Este é um método de ordenação não paramétrico, que plota as parcelas em um gráfico de dispersão, de forma que as distâncias euclidianas, entre as parcelas, são proporcionais à dissimilaridade entre elas (BABWETEERA e BROWN, 2009). As espécies com menos de 10 indivíduos foram eliminadas da análise, pois espécies raras acrescentam pouco em termos de informação, dificultando a interpretação dos dados. A adequabilidade da ordenação para interpretação foi avaliada por meio do valor de stress. As variáveis ambientais com maior significância $(p<0,001)$ sobre a florística e a estrutura do fragmento foram ajustadas, a posteriori, à ordenação produzida pela NMDS, por meio da função envfit. Para isso, foram utilizadas 1.000 permutações e excluídas da análise as variáveis de baixa significância e as redundantes. Encontradas as variáveis mais significativas, estas foram plotadas utilizando a função ordsurf, na forma de curvas de nível junto aos diagramas de ordenação, utilizando o ajuste produzido por um modelo aditivo generalizado, com thin plate splines. Todas as análises foram realizadas por meio do programa estatístico $R$ ( $R$ DEVELOPMENT CORE TEAM 2010), junto com a biblioteca Vegan (OKSANEN et al., 2009).

\section{RESULTADOS E DISCUSSÃO}

\section{Florística e diversidade}

Foram amostrados nas parcelas 1.843 
indivíduos arbóreos, que totalizaram uma área basal de $36,45 \mathrm{~m}^{2}$, distribuídos em 37 famílias, 63 gêneros e 92 espécies, sendo duas destas identificadas somente em nível de família e três em nível de gênero (Tabela 1). Além destas espécies, foram encontradas, fora das parcelas, mais seis espécies arbóreas (Tabela 1). As famílias de maior riqueza de espécies foram Myrtaceae (22), Lauraceae (sete), Aquifoliaceae (cinco), Asteraceae (cinco), Fabaceae (cinco) e Salicaceae (cinco). Os gêneros de maior riqueza foram Eugenia (cinco), Ilex (cinco), Myrcia (cinco) e Myrceugenia (quatro).

Comparando com outros trabalhos na região, com o mesmo nível de inclusão, a mesma intensidade amostral e na mesma bacia hidrográfica, a área basal encontrada no presente estudo $\left(36,45 \mathrm{~m}^{2} / \mathrm{ha}\right)$ foi semelhante ao de outro fragmento florestal estudado no município Lages, que obteve $35,9 \mathrm{~m}^{2} /$ ha (SILVA et al., dados não publicados), e a uma área de FOM Alto-Montana localizada no Município de Painel, SC (34,80 m²/ ha) (HIGUCHI et al., dados não publicados). Todos os valores de área basal supracitados podem ser considerados elevados de acordo a resolução $\mathrm{n}^{\mathrm{o}}$ 4 do CONAMA de 1994, que considera valores maiores do que $20 \mathrm{~m}^{2} / \mathrm{ha}$ como sendo indicativo de uma formação primária para as florestas do bioma atlântico, no Estado de Santa Catarina. A densidade (1.843 ind./ha) também foi semelhante ao fragmento estudado por Silva et al. (dados não publicados), que obteve 1.798 ind./ha, e superior ao $f$ ragmento localizado no município de Painel, com $1.395 \mathrm{ind} . / \mathrm{ha}$.

O perfil florístico da área estudada, com expressiva riqueza de espécies dos gêneros Myrcia, Ilex, Eugenia e Myrceugenia, está dentro do contexto encontrado em outros remanescentes de Floresta Ombrófila Mista localizados na região do Alto-Uruguai (HIGUCHI et al., dados não publicados). As famílias botânicas Myrtaceae e Salicaceae também se destacaram em elevada riqueza de espécies em outros estudos na região sul do Brasil (NASCIMENTO et al., 2001; SEGER et al., 2005; KOZERA et al., 2006; LINGNER et al., 2007), de forma que esse parece ser o padrão comum das florestas sulinas.

TABELA 1: Listagem florística do componente arbóreo do fragmento estudado de Floresta Ombrófila Mista Montana em Lages, Santa Catarina. * Espécies encontradas fora das parcelas.

TABLE 1: Tree component floristic list of the study of a montane Araucaria forest fragment, in Lages, Santa Catarina state. * Species found outside the plots.

\begin{tabular}{|c|c|}
\hline Família/Espécie & Família/Espécie \\
\hline ANACARDIACEAE & MYRTACEAE \\
\hline Lithraea brasiliensis Marchand & Acca sellowiana (O.Berg) Burret \\
\hline Schinus lentiscifolius Marchand* & Blepharocalyx salicifolius (Kunth) O.Berg \\
\hline Schinus polygamus (Cav.) Cabrera* & Calyptranthes concinna DC. \\
\hline Schinus terebinthifolius Raddi & Campomanesia xanthocarpa O.Berg \\
\hline ANNONACEAE & Eugenia pluriflora DC. \\
\hline Annona rugulosa (Schltdl.) H.Rainer & Eugenia pyriformis Cambess. \\
\hline AQUIFOLIACEAE & Eugenia sp. \\
\hline Ilex brevicuspis Reissek & Eugenia uniflora $\mathrm{L}$. \\
\hline Ilex dumosa Reissek & Eugenia uruguayensis Cambess. \\
\hline Ilex microdonta Reissek & Myrceugenia euosma (O.Berg) D.Legrand \\
\hline Ilex paraguariensis A.St.-Hil. & Myrceugenia myrcioides (Cambess.) O.Berg \\
\hline Ilex theezans Mart. Ex Reissek & Myrceugenia ovata (Hook. \& Arn.) O.Berg* \\
\hline ARALIACEAE & Myrceugenia oxysepala (Burret) D.Legrand \& Kausel \\
\hline Oreopanax fulvus Marchal & Myrcia guianensis (Aubl.) DC. \\
\hline ARAUCARIACEAE & Myrcia hatschbachii D.Legrand \\
\hline Araucaria angustifolia (Bertol.) Kuntze & Myrcia laruotteana Cambess. \\
\hline ASTERACEAE & Myrcia multiflora (Lam.) DC. \\
\hline Dasyphyllum spinescens (Less.) Cabrera & Myrcia palustris DC. \\
\hline Dasyphyllum tomentosum (Spreng.) Cabrera & Myrcianthes gigantea (D.Legrand) D.Legrand \\
\hline Gochnatia polymorpha (Less.) Cabrera & Myrciaria sp. \\
\hline
\end{tabular}


TABELA 1: Continuação ...

TABLE 1: Continued ...

\begin{tabular}{|c|c|}
\hline Família/Espécie & Família/Espécie \\
\hline Piptocarpha angustifolia Dusén & Myrrhinium atropurpureum Schott \\
\hline Vernonanthura discolor (Spreng.) H.Rob. & Myrtaceae não identificada \\
\hline BIGNONIACEAE & PODOCARPACEAE \\
\hline Handroanthus albus (Cham.) Mattos & Podocarpus lambertii Klotzsch ex Endl. \\
\hline Jacaranda puberula Cham. & PRIMULACEAE \\
\hline CANELLACEAE & Myrsine coriacea (Sw.) Roem. \& Schult. \\
\hline Cinnamodendron dinisii Schwacke & Myrsine sp. \\
\hline CANNABACEAE & Myrsine umbellata Mart. \\
\hline Celtis iguanaea (Jacq.) Sarg. & PROTEACEAE \\
\hline CARDIOPTERIDACEAE & Roupala montana Aubl. \\
\hline Citronella paniculata (Mart.) R.A.Howard & QUILLAJACEAE \\
\hline CELASTRACEAE & Quillaja brasiliensis (A.St.-Hil. \& Tul.) Mart. \\
\hline Maytenus boaria Molina & RHAMNACEAE \\
\hline Maytenus dasyclada Mart. & Rhamnus sphaerosperma Sw. \\
\hline CLETHRACEAE & Scutia buxifolia Reissek \\
\hline Clethra scabra Pers. & ROSACEAE \\
\hline CUNONIACEAE & Prunus myrtifolia (L.) Urb. \\
\hline Lamanonia ternata Vell. & RUBIACEAE \\
\hline DICKSONIACEAE & Coutarea hexandra (Jacq.) K.Schum. \\
\hline Dicksonia sellowiana Hook. & RUTACEAE \\
\hline ERYTHROXYLACEAE & Zanthoxylum kleinii (R.S.Cowan) P.G.Waterman \\
\hline Erythroxylum deciduum A.St.-Hil. & Zanthoxylum rhoifolium Lam. \\
\hline ESCALLONIACEAE & SALICACEAE \\
\hline Escallonia bifida Link. \& Otto & Banara tomentosa Clos \\
\hline EUPHORBIACEAE & Casearia decandra Jacq. \\
\hline Sebastiania commersoniana (Baill.) L.B.Sm. \& & Casearia obliqua Spreng. \\
\hline Downs. & Xylosma ciliatifolia (Clos) Eichler \\
\hline Sapium glandulosum (L.) Morong & Xylosma tweediana (Clos) Eichler \\
\hline Sebastiania brasiliensis Spreng. & SAPINDACEAE \\
\hline FABACEAE & Allophylus edulis (A.St.-Hil., Cambess. \& A.Juss.) Radlk. \\
\hline Dalbergia frutescens (Vell.) Britton & Allophylus guaraniticus (A.St.-Hil.) Radlk. \\
\hline Inga sessilis (Vell.) Mart. & Cupania vernalis Cambess. \\
\hline Machaerium paraguariense Hassl. & Matayba elaeagnoides Radlk. \\
\hline Machaerium stipitatum (DC.) Vogel & SOLANACEAE \\
\hline Mimosa scabrella Benth. & Solanum pabstii L.B.Sm. \& Downs \\
\hline LAURACEAE & Solanum sanctaecatharinae Dunal \\
\hline Cinnamomum amoenum (Nees \& Mart.) Kosterm. & STYRACACEAE \\
\hline Lauraceae não identificada & Styrax leprosus Hook. \& Arn. \\
\hline Nectandra lanceolata Nees & SYMPLOCACEAE \\
\hline Nectandra megapotamica (Spreng.) Mez & Symplocos uniflora (Pohl) Benth. \\
\hline Ocotea diospyrifolia (Meisn.) Mez & THYMELAEACEAE \\
\hline Ocotea puberula (Rich.) Nees & Daphnopsis racemosa Griseb.* \\
\hline Ocotea pulchella Mart. & VERBENACEAE \\
\hline MELASTOMATACEAE & Duranta vestita Cham. \\
\hline Miconia hiemalis A.St.-Hil. \& Naudin ex Naudin & WINTERACEAE \\
\hline MELIACEAE & Drimys brasiliensis Miers \\
\hline \multicolumn{2}{|l|}{ Cabralea canjerana (Vell.) Mart. } \\
\hline Cedrela fissilis Vell. & \\
\hline
\end{tabular}


$\mathrm{O}$ índice de diversidade de Shannon (H') foi de 3,74 e a equabilidade de Pielou (J) de 0,83. $\mathrm{O}$ valor encontrado de $\mathrm{H}^{\prime}$ pode ser considerado elevado, quando comparado com outros trabalhos realizados em fragmentos de FOM, na região Sul do Brasil, como o realizado por Nascimento et al. (2001) $\left(H^{\prime}=3,0\right.$ para o nível de inclusão de diâmetro medido à altura do peito, $\mathrm{DAP} \geq 9,6 \mathrm{~cm}$ ). A equabilidade pode ser considerada igualmente elevada, o que, segundo Souza et al. (2003), indica que existe pouca concentração de abundância em espécies dominantes. A riqueza total encontrada, incluindo as espécies identificadas até o nível de família e gênero, de 98 espécies, correspondeu, respectivamente, a 89,42 e $80,18 \%$ da riqueza esperada, de acordo com os estimadores de Jackknife de primeira $(109,6)$ e segunda ordem $(122,22)$. O número de espécies arbóreas encontrado (98) pode ser considerado elevado quando comparado com outros levantamentos de fragmentos de FOM, na região sul do Brasil, que variaram de $39 \mathrm{em}$ Clevelândia (WATZLAWICK et al., 2009), PR, a 125 em Caxias do Sul (RAMOS e BOLDO, 2007), RS. A riqueza de espécies arbóreas encontrada no fragmento estudado correspondeu a 73,68\% da riqueza encontrada por Vibrans et al. (2008) para a área de FOM, no inventário florístico-estrutural do Estado de Santa Catarina, considerando uma amostragem de 14,8 ha e um nível de inclusão de DAP $\geq 10 \mathrm{~cm}$. O elevado número de espécies encontrado pode ser uma resposta a elevada heterogeneidade ambiental existente.

\section{Estrutura horizontal}

Das dez espécies com os maiores valores de importância, destacaram-se Araucaria angustifolia, Lithraea brasiliensis Marchand, Jacaranda puberula Cham. e Casearia decandra Jacq. (Tabela 2). Araucaria angustifolia se destacou como a espécie de maior dominância devido a uma grande quantidade de indivíduos com elevado DAP. Lithraea brasiliensis se destacou pela maior frequência na área, demonstrando uma ampla distribuição. Jacaranda puberuala se destacou como a espécie com maior densidade. Casearia decandra também apresentou alta densidade (segunda espécie com maior densidade) e ampla ocorrência na área, porém, apresentou uma área basal de apenas 1,19 $\mathrm{m}^{2}$, com predominância de indivíduos pequenos, confirmando o estudo de Kozera et al. (2006), no Paraná, quando consideraram esta espécie como um importante componente do sub-bosque da floresta. Um total de 20 espécies apresentou apenas um indivíduo amostrado, o que representou $22,22 \%$ do total das espécies encontradas.

Araucaria angustifolia foi a primeira espécie no ranking de importância em outros estudos realizados na região (RONDON NETO et al., 2002; SEGER et al., 2005; LINGNER et al., 2007). Matayba elaeagnoides Radlk. (NASCIMENTO et

TABELA 2: Parâmetros fitossociológicos das dez espécies arbóreas de maior valor de importância (VI, em\%) do fragmento estudado de Floresta Ombrófila Mista Montana, em Lages, SC. DA = densidade absoluta (ind./ha); $\mathrm{DR}=$ densidade relativa (\%); DoA = dominância absoluta $\left(\mathrm{m}^{2} / \mathrm{ha}\right) ; \mathrm{DoR}=$ dominância relativa (\%); FA = frequência absoluta (\%); FR = frequência relativa (\%).

TABLE 2: Phyto-sociological parameters of the ten tree species with the highest importance value (VI\%) present in the study of a montane Araucaria forest fragment in Lages, Santa Catarina state. AD = absolute density (ind./ha), DR = relative density (\%); DoA = absolute dominance $\left(\mathrm{m}^{2} / \mathrm{ha}\right) ;$ DoR $=$ relative dominance $(\%) ; \mathrm{FA}=$ absolute frequency $(\%), \mathrm{RF}=$ relative frequency $(\%)$.

\begin{tabular}{lccccccc}
\hline \multicolumn{1}{c}{ Espécies } & DA & DR & DoA & DoR & FA & FR & VI \\
\hline Araucaria angustifolia & 125 & 6,78 & 5,27 & 14,46 & 68 & 4,05 & 8,43 \\
Lithraea brasiliensis & 101 & 5,48 & 3,94 & 10,80 & 80 & 4,76 & 7,01 \\
Jacaranda puberula & 172 & 9,33 & 1,54 & 4,23 & 46 & 2,74 & 5,43 \\
Casearia decandra & 149 & 8,08 & 1,19 & 2,53 & 86 & 5,12 & 5,48 \\
Matayba elaeagnoides & 84 & 4,56 & 2,21 & 6,05 & 38 & 2,26 & 4,29 \\
Podocarpus lambertii & 69 & 3,74 & 1,48 & 4,05 & 64 & 3,81 & 3,87 \\
Sapium glandulosum & 66 & 3,58 & 1,26 & 3,45 & 40 & 2,38 & 3,14 \\
Ocotea pulchella & 34 & 1,84 & 1,60 & 4,40 & 52 & 3,10 & 3,11 \\
Myrsine umbellata & 69 & 3,74 & 0,70 & 1,93 & 56 & 3,33 & 3,00 \\
Lamanonia ternata & 37 & 2,01 & 1,67 & 4,58 & 40 & 2,38 & 2,99 \\
\hline
\end{tabular}


Higuchi, P. et al.

al., 2001; FORMENTO et al., 2004), Lamanonia ternata Vell. (LINGNER et al., 2007), Ocotea pulchella Mart. (FORMENTO et al., 2004) e Lithraeae brasiliensis (NASCIMENTO et al., 2001; RONDON NETO et al., 2002; FORMENTO et al., 2004; SEGER et al., 2005) também são frequentemente encontradas entre as dez espécies de maior VI em remanescentes de FOM na região sul do Brasil. Araucaria angustifolia se destaca nos fragmentos de FOM em função de suas copas típicas dominantes. No entanto, existe um conjunto de espécies angiospérmicas de ampla distribuição geográfica e importante contribuição na comunidade florestal, como as citadas acima, que também se destacam ocupando o espaço vertical abaixo das copas dos indivíduos de araucária. Desta forma, pode-se considerar que a fitofisionomia da FOM é caracterizada não apenas pela presença marcante de araucária, que já é um senso comum, mas também por um conjunto importante de espécies folhosas típicas.

\section{Variáveis ambientais}

Os valores médios de nutrientes nos solos (Tabela 3), de acordo com o critério de definição do substrato proposto por Oliveira Filho (2009), indicou um solo de fertilidade intermediária, pois o valor médio de saturação de base encontrado $(44,61 \%)$ foi de um solo mesonútrico. De acordo com a classificação proposta por Souza (2007), apesar de a declividade média indicar uma área com relevo ondulado $\left(11,83^{\circ}\right)$, existiu uma grande variação no fragmento, desde locais com a topografia suave ondulada $\left(4,5^{\circ}\right)$ até forte ondulada $\left(37,85^{\circ}\right)$. A cobertura do dossel, quando comparado com outro fragmento de Floresta Ombrófila Mista (60\%) (MEDRI et al., 2009), que utilizou a mesma metodologia de avaliação, pode ser considerada alta.

A PCA permitiu verificar que as variáveis ambientais não se distribuem de forma homogênea na área (Figura 3). Pode ser observado que os vetores que representam o $\mathrm{pH}(\mathrm{ph})$, a saturação de base $(\mathrm{V})$, a soma de base $(\mathrm{SB})$, o sódio $(\mathrm{Na})$, o potássio $(\mathrm{K})$, o cálcio $(\mathrm{Ca})$, o magnésio $(\mathrm{Mg})$, o fósforo $(\mathrm{P})$, a matéria orgânica $(\mathrm{MO})$, a declividade média (decmed) e o desnível máximo (desmax) apontaram para o lado direito do gráfico. Os vetores que representam o alumínio (Al), o alumínio mais hidrogênio (hal), a CTC efetiva (CTCef), a CTC a pH 7 (CTCph7), a cota média (cotmedia) e a cobertura do dossel (CD) apontaram para o lado
TABELA 3: Valores médios das variáveis ambientais analisadas em 50 parcelas alocadas no fragmento estudado de Floresta Ombrófila Mista Montana, em Lages, SC.

TABLE 3: Mean values of environmental variables analyzed within 50 plots allocated in the study of a montane Araucaria forest fragment in Montana Lages, Santa Catarina state.

\begin{tabular}{lcc}
\hline \multicolumn{1}{c}{ Variáveis } & Média & Desvio Padrão \\
\hline Argila $(\%)$ & 26,68 & 5,56 \\
$\mathrm{pH}$ & 4,87 & 0,61 \\
$\mathrm{P}(\mathrm{mg} / \mathrm{dm})$ & 6,05 & 4,51 \\
$\mathrm{M.O.}(\%)$ & 8,11 & 2,16 \\
$\mathrm{H}+\mathrm{Al}\left(\mathrm{cmolc} / \mathrm{dm}^{3}\right)$ & 12,32 & 5,90 \\
$\mathrm{Al}^{3+}$ & 2,07 & 2,59 \\
$\mathrm{Ca}^{2+}\left(\mathrm{cmolc}_{\mathrm{dm}}\right)$ & 6,99 & 2,84 \\
$\mathrm{Na}$ & 2,24 & 3,56 \\
$\mathrm{Mg}{ }^{2+}\left(\mathrm{cmolc} / \mathrm{dm}^{3}\right)$ & 1,91 & 1,27 \\
$\mathrm{~K}(\mathrm{mg} / \mathrm{dm})$ & 135,78 & 87,81 \\
$\mathrm{~V}(\%)$ & 44,61 & 19,03 \\
$\mathrm{SB}\left(\mathrm{cmolc} / \mathrm{dm}^{3}\right)$ & 9,27 & 3,72 \\
$\mathrm{CTC}$ efetiva $\left(\mathrm{cmolc} / \mathrm{dm}^{3}\right)$ & 23,66 & 5,65 \\
$\mathrm{CTC}$ a pH $=7\left(\mathrm{cmolc} / \mathrm{dm}^{3}\right)$ & 21,58 & 4,03 \\
Declividade média (graus) & 11,83 & 7,70 \\
Desnível máximo (m) & 6,22 & 3,80 \\
Cota média (m) & $1.000,54$ & 41,46 \\
Cobertura do Dossel (\%) & 87,20 & 6,55 \\
\hline
\end{tabular}

esquerdo. Dessa forma, é possível observar uma dicotomia nas características ambientais estudadas, podendo as parcelas estudadas serem classificadas em dois grupos distintos: locais com solos menos ácidos, mais férteis, de topografia mais inclinada e de menores altitudes, representados pelas parcelas que ocorrem no lado direito do diagrama de ordenação, e locais com solos mais ácidos, maior saturação por alumínio, de topografia mais plana, de maior altitude e com maior cobertura do dossel, representados pelas parcelas que ocorrem no lado esquerdo.

A análise NMDS (Figura 4) obteve valor de stress de 15,03\%, indicando que os diagramas de ordenação construídos são adequados para interpretação, de forma que a distância euclidiana das áreas nos diagramas está correlacionada de forma negativa com a similaridade florística entre elas. É possível observar que o componente arbóreo apresentou diferenciação florística e estrutural 


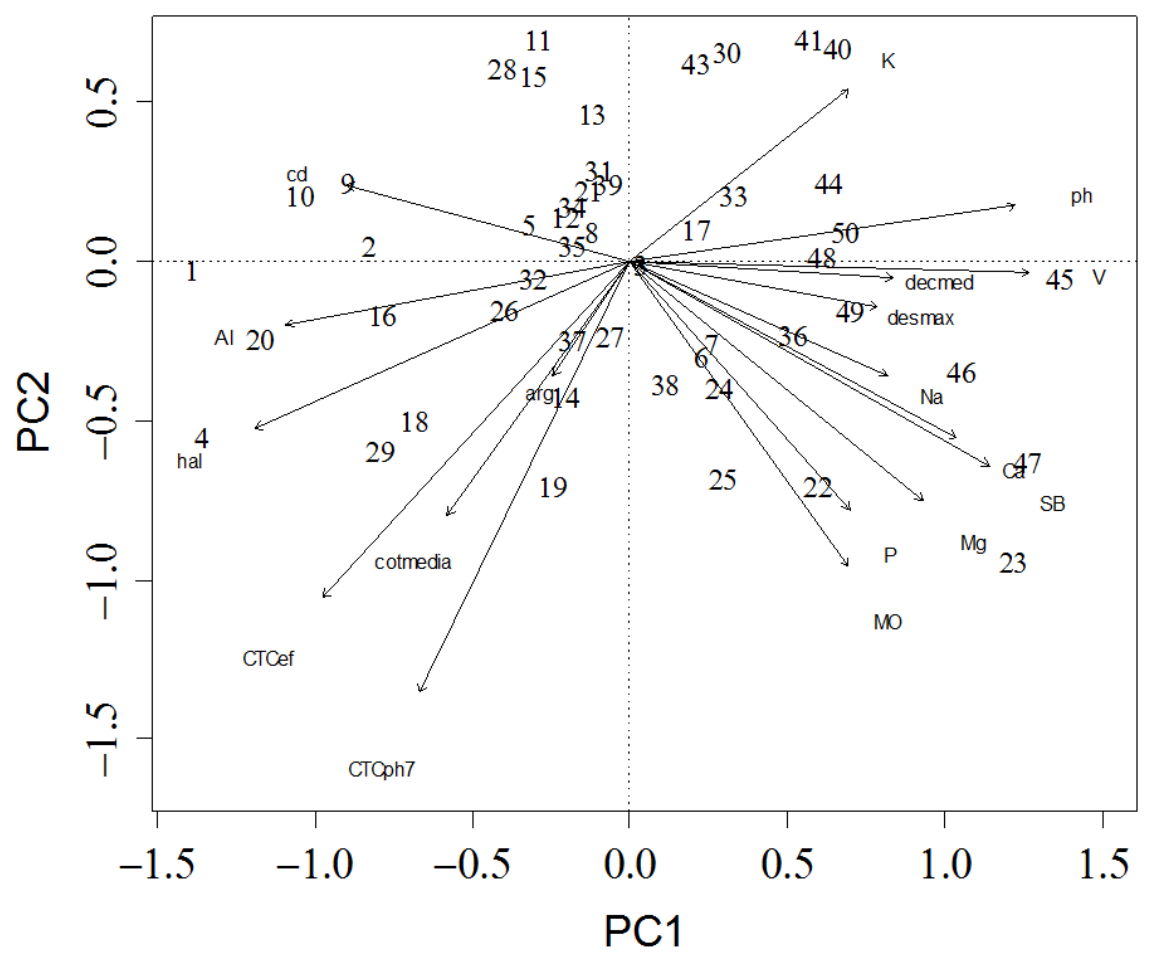

FIGURA 3: Distribuição das parcelas em uma análise de componentes principais (PCA) realizada para as variáveis ambientais coletadas no fragmento estudado de Floresta Ombrófila Mista Montana em Lages, SC (cd: cobertura do dossel, Al: Alumínio, hal: H + Al, CTCef: CTC efetiva, cotmedia: Cota média, CTCph7: CTC a ph 7, MO: matéria orgânica, P: fósforo, Mg: magnésio, Ca: cálcio, SB: soma de bases, Na: sódio, desmax: desnível máximo, decmed: declividade média, V: saturação por base, $\mathrm{ph}: \mathrm{pH}, \mathrm{K}$ : potássio).

FIGURE 3: Plots distribution in a principal component analysis (PCA) performed for environmental variables collected in a montane Araucaria forest fragment in Lages, Santa Catarina state (cd: canopy closure, Al: Aluminum, hal: $\mathrm{H}+\mathrm{Al}$, CTCef: effective CTC, cotmedia: mean elevation CTCph7: CTC at pH 7, MO: organic matter, P: phosphorus, Mg: magnesium, Ca: calcium , SB: sum of bases, Na: sodium, desmax: maximum height difference, decmed: mean declivity, $\mathrm{V}$ : base saturation, ph: $\mathrm{pH}, \mathrm{K}$ : potassium).

fortemente associada $(p<0,001)$ às variáveis químicas do solo ( $\mathrm{pH}$ e Mg) e à topográfica (desnível máximo da parcela). De acordo com a análise, as parcelas 44,45 e 50 formaram um agrupamento florístico associado a áreas com maiores valores de $\mathrm{pH}(>5,6)$ e de teores de $\mathrm{Mg}(>3,8)$ (Figuras 4A e 4C). Schinus terebinthifolius Raddi, Matayba elaeagnoides e Allophylus guaraniticus (A.St.-Hil.) Radlk. foram as espécies que melhor representam este agrupamento (Figuras 4B e 4D). Nos locais com baixos valores de $\mathrm{pH}(<4,6)$, ocorreu a formação de um agrupamento coeso, demonstrado pela curta distância das parcelas no diagrama, com um grande número de parcelas $(2,3,4,5,6,8,9,10,13,17,21$, 25 e 32). As espécies que melhor caracterizam este agrupamento foram Calyptranthes concinna DC., Zanthoxylum kleinii (R.S.Cowan) P.G.Waterman,
Solanum sanctaecatharinae Dunal, Jacaranda puberula e Araucaria angustifolia. Nos locais com os menores valores de $\mathrm{Mg}(<1,2)$ ocorreu o agrupamento das parcelas 10,28, 29, 30, 33 e 34, com Casearia decandra se destacando.

No diagrama que demonstra a relação das parcelas e espécies com o desnível máximo, nos locais com maior desnível máximo (>11 m) e maior declividade, ocorreu o agrupamento das parcelas 45, 46, 47, 48 e 49 (Figura 4E). Nestas parcelas, houve maior adensamento de Allophylus guaraniticus (Figura 4F). No outro extremo do gradiente, nos locais mais planos, com desnível máximo inferior a $4 \mathrm{~m}$, ocorreu o agrupamento das parcelas $2,3,4$, $6,8,25$ e 32, com maior densidade das espécies Calyptranthes concinna, Solanum sanctacatarine e Jacaranda puberula. 

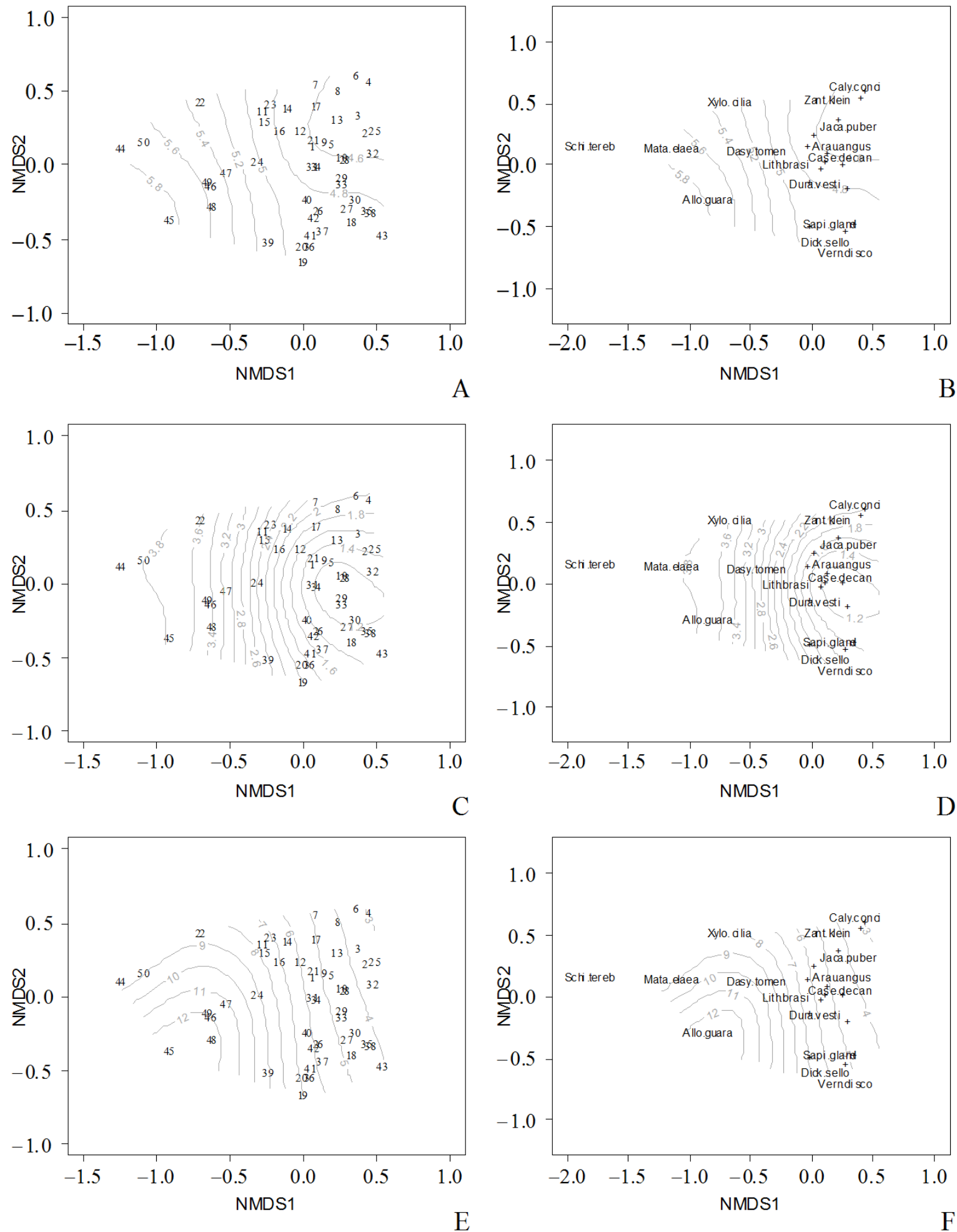

FIGURA 4: Diagramas de ordenação das parcelas (coluna da esquerda) e espécies (coluna da direita), produzidos pela análise NMDS (Nonmetric multidimensional scalling) no fragmento estudado de Floresta Ombrófila Mista Montana localizado em Lages, SC, e variáveis ambientais plotadas $(p<0,001)$ : $\mathrm{pH}$ (A e B) magnésio (C e D) e desnível máximo (E e F).

FIGURE 4: Ordination diagrams of plots (left column) and species (right column) produced by NMDS analysis (Nonmetric Multidimensional Scaling) analyzed in the study of a montane Araucaria forest fragment in Lages, Santa Catarina state, and environmental variable plotted $(p<0.001)$ : $\mathrm{pH}(\mathrm{A}$ and $\mathrm{B})$ magnesium $(\mathrm{C}$ and $\mathrm{D})$ and highest declivity (E and $\mathrm{F})$. 


\section{CONCLUSÕES}

$\mathrm{O}$ presente estudo demonstrou que $\mathrm{o}$ fragmento florestal estudado apresenta uma composição florística e a estrutura semelhante a outros fragmentos florestais da região. $\mathrm{O}$ componente arbóreo apresentou variações espaciais que refletem a heterogeneidade ambiental existente. Os resultados reforçam a ideia de que o planejamento de estratégias de restauração florestal deve considerar as variações da vegetação, em função das condições ambientais existentes.

\section{AGRADECIMENTOS}

Ao CNPq, pelo auxílio financeiro (Edital $\mathrm{MCT} / \mathrm{CNPq}$ 15/2007). Ao Sr. Jorge Gamborgi, por ter gentilmente permitido a realização do presente trabalho em suas propriedades.

\section{REFERÊNCIAS BIBLIOGRÁFICAS}

APG III. An update of the Angiosperm Phylogeny Group classification for the orders and families of flowering plants: APG III. Botanical Journal of the Linnean Society, v. 161, n. 2, p. 105-121. 2009. BABWETEERA, F.; BROWN, N. Can remnant frugivore species effectively disperse tree seeds in secondary tropical rain forests? Biodiversity and Conservation, v. 18, n. 6, p. 1611-1627. 2009.

BROWER, J.; ZAR, J.; VON ENDE, C. Field and laboratory methods for general ecology. Dubuque: McGraw-Hill Science, 1990. 161 p.

CARDOSO, E.; SCHIAVINI, I. Relação entre distribuição de espécies arbóreas e topografia em um gradiente florestal na Estação Ecológica do Panga (Uberlândia, MG). Revista Brasileira de Botânica, v. 25, n. 3, p. 277-289. 2002.

EMBRAPA. Manual de métodos de análises de solo. 2. ed. Rio de Janeiro: Centro Nacional de Pesquisa de Solos, 1997. 247 p.

ESPÍRITO-SANTO, F. et al. Variáveis ambientais ea distribuição de espécies arbóreas em um remanescente de floresta estacional semidecídua montana no campus da Universidade Federal de Lavras, MG. Acta Botanica Brasilica, v. 16, n. 3, p. 331-356. 2002.

FORMENTO, S.; SCHORN, L.; RAMOS, R. Dinâmica estrutural arbórea de uma Floresta Ombrófila Mista em Campo Belo do Sul, SC. Cerne, v. 10, n. 2, p. 196-212. 2004.

HELTSHE, J.; FORRESTER, N. Estimating species richness using the jackknife procedure. Biometrics, v. 39, n. 1, p. 1-11. 1983.

HIGUCHI, P. et al. Dinâmica da comunidade arbórea em um fragmento de floresta estacional semidecidual montana em Lavras, Minas Gerais, em diferentes classes de solos. Revista Árvore, v. 32, n. 3, p. 417-426. 2008a.

HIGUCHI, P. et al. Spatio-temporal patterns of tree community dynamics in a tropical forest fragment in South-east Brazil. Plant Ecology, v. 199, n. 1, p. 125-135. 2008b.

HIJMANS, R. et al. Very high resolution interpolated climate surfaces for global land areas. International Journal of Climatology, v. 25, n. 15, p. 1965-1978. 2005.

IBGE. Manual técnico da vegetação brasileira. Série: Manuais técnicos em geociências n.1. Rio de Janeiro: Fundação Instituto Brasileiro de Geografia e Estatística, 1992. 92 p.

KOZERA， C.; DITTRICH, V.; SILVA, S. Fitossociologia do componente arbóreo de um fragmento de Floresta Ombrófila Mista Montana, Curitiba, PR, BR. Floresta, v. 36, n. 2, p. 225-237. 2006.

LEMMON, P. A spherical densiometer for estimating forest overstory density. Forest Science, v. 2, n. 4, p. 314-320. 1956.

LINGNER, D. et al. Caracterização da estrutura e da dinâmica de um remanescente de Floresta com Araucária no Planalto Catarinense. Pesquisa Florestal Brasileira, n. 55, p. 55-66. 2007.

MEDRI, P. et al. Comparação de parâmetros bióticos e abióticos entre fragmento de floresta secundária e reflorestamento de Araucaria angustifolia (Bertol.) O. Kuntze. Semina: Ciências Biológicas e da Saúde, v. 30, n. 2, p. 185-194. 2009.

MINCHIN, P. An evaluation of the relative robustness of techniques for ecological ordination.

Plant Ecology, v. 69, n. 1, p. 89-107. 1987.

MUELLER-DOMBOIS, D.; ELLEMBERG, $\mathrm{H}$. Aims and methods of vegetation ecology. New York: John Wiley, 1974. $547 \mathrm{p}$.

NASCIMENTO, A.; LONGHI, S.; BRENA, D. Estrutura e padrões de distribuição espacial de espécies arbóreas em uma amostra de Floresta Ombrófila Mista em Nova Prata, RS. Ciência Florestal, Santa Maria, v. 11, n. 1, p. 105-119. 2001. OKSANEN, J. et al. Vegan: community ecology package. $\mathbf{R}$ package version, v. 1, p. 8-8. 2009.

OLIVEIRA FILHO, A. T. Classificação das fitofisionomias da América do Sul cisandina tropical e subtropical: proposta de um novo sistema- 
prático e flexível-ou uma injeção a mais de caos. Rodriguésia, v. 60, n. 2, p. 237-258. 2009.

OLIVEIRA FILHO, A. T. et al. Differenciation of streamside and upland vegetation in an area of montane semideciduous Forest in southeastern Brasil. Flora, v. 189, p. 1-19. 1994.

OLIVEIRA FILHO, A. T. et al. Dinâmica da comunidade e populações arbóreas da borda e interior de um remanescente florestal na Serra da Mantiqueira, Minas Gerais, em um intervalo de cinco anos (1999-2004). Revista Brasileira de Botânica, v. 30, n. 1, p. 149-161, 2007.

OLIVEIRA FILHO, A. T.; MELLO, J.; SCOLFORO, J. R. Effects of past disturbance and edges on tree community structure and dynamics within a fragment of tropical semideciduous forest in south-eastern Brazil over a five-year period (1987-1992). Plant Ecology, v. 131, n. 1, p.45-66. 1997.

PUCHALSKI, Â. Variações edafo-climáticas e ocorrência natural de Araucaria angustifolia (Bert.) O. Kuntze no Estado de Santa Catarina. 2004. 85 f. Dissertação (Mestrado em Recursos Genéticos Vegetais)-Universidade Federal de Santa Catarina, Florianópolis, 2004.

R DEVELOPMENT CORE TEAM. R: A language and environment for statistical computing. $\mathrm{R}$ Foundation for Statistical Computing, Vienna, Disponível em: $<$ (http://www.R-project.org) $>$ Acesso em: 16 de março de 2010.

RAMOS, A. J. K.; BOLDO, E. Diversidade florística e aspectos fitossociológicos de formações florestais em estágio sucessional secundário na Floresta Ombrófila Mista, município de Caxias do Sul-RS. Revista Brasileira de Agroecologia, v. 2, n. 1, p. 111-116. 2007.

RODRIGUES, L. et al. Efeitos de solos e topografia sobre a distribuição de espécies arbóreas em um fragmento de floresta estacional semidecidual.
Luminárias, MG. Revista Árvore, v. 31, n. 1, p. 25-35. 2007.

RONDON NETO, R. et al. Caracterização florística e estrutural de um fragmento de floresta ombrófila mista em Curitiba, PR, Brasil. Floresta, v. 32, n. 1, p. 3-16. 2002.

SEGER, C. et al. Levantamento florístico e análise fitossociológica de um remanescente de floresta ombrófila mista localizado no município de Pinhais, Paraná-Brasil. Floresta, v. 35, n. 2, p. 291-302. 2005.

SILVA, A. C. et al. Florística e estrutura da comunidade arbórea em fragmentos de floresta aluvial em São Sebastião da Bela Vista, Minas Gerais, Brasil. Revista Brasileira de Botânica, v. 32, n. 2, p. 283-297. 2009.

SILVA, A. C.; HIGUCHI, P.; VAN DEN BERG, E. Effects of soil water table regime on tree community species richness and structure of alluvial forest fragments in Southeast Brazil. Brazilian Journal of Biology, v. 70, n. 3, p. 465-471, 2010.

SOUZA, C. G. Manual técnico de pedologia. Série: Manuais Técnicos em Geociências v. 4. Rio de Janeiro: Fundação Instituto Brasileiro de Geografia e Estatística, 2007. 104 p.

SOUZA, J. S. et al. Análises das variações florísticas e estruturais da comunidade arbórea de um fragmento de floresta semidecídua às margens do rio Capivari, Lavras-MG. Revista Árvore, v. 27, n. 2, p. 185-206, 2003.

VIBRANS, A. et al. Ordenação dos dados de estrutura da Floresta Ombrófila Mista partindo de informações do inventário florístico-florestal de Santa Catarina: resultados de estudo-piloto. Ciência Florestal, v. 18, n. 4, p. 511-523. 2008.

WATZLAWICK, L. et al. Caracterização da composição florística e estrutura de uma Floresta Ombrófila Mista, no município de General Carneiro (PR). Ambiência, v. 1, n. 2, p. 229-237. 2009. 